Discussion Paper Series No. 187

Indeterminacy in the free-trade world

\begin{tabular}{ll} 
DOI Junko & $\begin{array}{l}\text { Kansai University/ } \\
\text { Kyoto Sangyo University }\end{array}$ \\
\hline IWASA Kazumichi & Kobe University \\
SHIMOMURA Koji & Kobe University
\end{tabular}

May 2006

Discussion papers are a series of manuscripts in their draft form. They are not intended for circulation or distribution except as indicated by the author. For that reason Discussion Papers may not be quoted, reproduced or distributed without the written consent of the author. 


\title{
Indeterminacy in the free-trade world
}

\author{
Junko Doi \\ Kansai University/Kyoto Sangyo University \\ Kazumichi Iwasa \\ Koji Shimomura* \\ GSE, Kobe University \\ RIEB, Kobe University
}

May 10, 2006

\begin{abstract}
We show that indeterminacy arises in a discrete-time competitive twocountry dynamic model of international trade in which externalities, imperfect competition, public goods, and government intervention are assumed away. The present model is a standard dynamic trade model in the sense that there is neither an international credit market nor international factor mobility, and these intrinsic features are a source of indeterminacy. Indeterminacy is implied by the condition for the existence of a steady state.
\end{abstract}

Acknowledgement 1 We are grateful to Satya Das, Kazuo Mino, and Stephen J. Turnovsky for their insightful comments and suggestions.

\section{Introduction}

Recently, researchers majoring in economic dynamics have paid much attention to indeterminacy which is defined as the existence of a continuum of dynamic equilibrium paths starting from historically given initial conditions of state variables and converging to a common steady state ${ }^{1}$ : It is indeterminate in a decentrarized market economy which equilibrium path is realized. Kazuo Nishimura is a leading academic economist who has made considerable contributions to this topic: joint works with Jess Benhabib and Qinglai Meng (Benhabib and Nishimura (1998) and Benhabib, Meng and Nishimura (2000)) have made path-breaking findings concerning indeterminacy in multi-sector dynamic general equilibrium frameworks of exogenous and endogenous growth in which returns to scale are socially constant but privately decreasing. ${ }^{2}$

\footnotetext{
*Corresponding author: RIEB, Kobe University, 2-1 Rokkodai-cho, Nada-ku, Kobe, Japan, 657-8501: Tel. 81-78-803-7002:. Fax.81-78-803-7059: E-mail. simomura@rieb.kobe-u.ac.jp.

${ }^{1}$ See, for example, Benhabib and Farmer (1994, 1996, 1999), Boldrin and Rustichini (1994), Mino (2001), and Nishimura and Shimomura (2002a).

${ }^{2}$ See also Nishimura, Shimomura and Wang (2006) and Mino Nishimura, Shimomura and Wang (2005).
} 
His another notable contribution to this topic is that while almost all contributions to the literature on indeterminacy assumes a closed economy, his joint work with one of the authors of this paper (Nishimura and Shimomura $(2002 b))$ is the first to study indeterminacy in the standard two-country dynamic Heckscher-Ohlin model in which, using a standard trade-theory term, factor-generated external economies of scale are assumed. Nishimura and Shimomura (2002b) also discuss about a negative implication of indeterminacy for the factor endowment theory of international trade such that a small extent of factor-generated externalities may violate the long-run trade pattern predicted by the dynamic Heckscher-Ohlin theorem (Chen (1992)), a dynamic version of a fundamental theorem in trade theory.

Nishimura and Shimomura (2002b) followed the preceding literature on indeterminacy in assuming factor-generated externalities. Later, Nishimura and Shimomura (2005) find that, even if not only such externalities but also other market-distortional factors like imperfect competition, public goods. and trade policies are assumed away, indeterminacy is still possible in a dynamic continuoustime, free-trade, and two-country model based on the standard assumptions in trade theory such that (i) two commodities, a pure consumption good and a consumable capital are produced by using capital and labor under constantreturns-to-scale technologies, (ii) while commodities are freely traded among countries, factors of production are internationally immobile and (iii) there is no international credit market. ${ }^{3}$ Shimomura (2004) also obtains an indeterminacy result in a continuous-time dynamic two-country model in which there are two tradable goods, durable and non-durable consumption goods, both of which are produced by using only primary factors of production called labor whose stocks are constant over time.

Those two papers share a problem to get over; to guarantee the indeterminacy result, the sum of the rate of capital depreciation and the rate of time preference must be exactly equal between two countries; moreover, the domestic rate of time preference must be internationally different; however small the international difference in the sum may be, the indeterminacy result is not generally guaranteed. Such a restrictive condition is apparently undesirable.

In this paper, we construct a simple dynamic version of Ricardian model of international trade and derives an indeterminacy result without such severe restrictions on parameters. Moreover, we show that in the present dynamic model the existence of a steady state is sufficient for indeterminacy. That is, if there exists a steady state in the dynamic trade model, there must be multiple steady states such that there is a continuum of equilibrium paths starting from a neighborhood of one of the steady states. Moreover, the condition for the existence are less restrictive than the condition imposed in the above two papers in the sense that the former condition is no loner of "knife-edge" type.

This paper is organized as follows. Section 2 sets up the model. Section 3 discusses under what conditions a steady state exists and shows that if a

\footnotetext{
${ }^{3}$ Needless to say, there is a huge literature in international macroeconomics in which there is an international credit market. On the other hand, it is usually assumed away in a dynamic trade model which focuses on trade patterns.
} 
steady state exists, two non-degenerate steady states exist. Section 4 shows that there is a continuum of equilibrium paths starting from a stock of the durable consumption good which is sufficiently close to one of the two steady states. Section 5 makes some concluding remarks.

\section{The model}

The two-country model in this paper is a specific version of the dynamic trade model developed in Shimomura (1993). The trading world consists of two countries called India and US, and two goods are traded between the countries. Both goods are consumption goods, but one of them, say wheat, is a purely non-durable good while the other, say computer, is a durable good with a constant rate of depreciation; both goods are produced by using only labor with constant labor coefficients, $\left(a_{1}, a_{2}\right)$ in US and $\left(a_{1}^{*}, a_{2}^{*}\right)$ in India.

Let us begin with the explanation of optimal consumption plans of US and Indian households.

\subsection{Households}

Each US household supplies $L$ units of labor inelastically, while each Indian household supplies $L^{*}$ units of labor. The population of each country is normalized to be unity.

Concerning preferences, we assume, just for simplicity, that the representative US household derives utility from consuming wheat $c$ and operating computers $B$, while the representative household in India derives utility only from consuming wheat, say $c^{*}$.

\subsubsection{The US household}

We assume that the US felicity function, $u_{t}=U\left(c_{t}, B_{t}\right)$, is increasing and concave in $c_{t}$ and $B_{t}$, and satisfies the Inada conditions. The objective of the US household is to maximize the discounted sum of utility:

$$
\begin{gathered}
\max _{u_{t}, A_{t}} \sum_{t=0}^{\infty} u_{t} \rho^{t} \quad \text { subject to } \\
S_{t+1}=\left(1+r_{t}\right) S_{t}+w_{t} L-E\left(p_{t}, R_{t}, u_{t}\right), \quad S_{0} ; \text { given, }
\end{gathered}
$$

where $S_{t}$ the asset owned by a household at period $t$, which consists of the stock of computers $A_{t}$ and the net credit $F_{t}$ owned by the US household; the labor endowment of the household, $L$, is positive and time-invariant; $r_{t}$ the interest 
rate, $R_{t}$ the rental rate of computers, $w_{t}$ the wage rate, and $p_{t}$ the price of wheat in terms of computers; $\rho$ the discount factor and $\delta$ the rate of depreciation, both of which are positive, time-invariant, and in between 0 and 1 . We assume that one unit of computer generates one unit of service flow during each period. Computers serve as the numeraire.

$E\left(p_{t}, R_{t}, u_{t}\right)$ is called the period- $t$ expenditure function; we define it as

$$
E\left(p_{t}, R_{t}, u_{t}\right) \equiv \min _{c_{t}, B_{t}} p_{t} c_{t}+R_{t} B_{t} \text { subject to } u_{t} \leq U\left(c_{t}, B_{t}\right)
$$

The envelope properties of the expenditure function ensure us that the partial derivatives of $E\left(p_{t}, R_{t}, u_{t}\right)$ with respect to $p_{t}$ and $R_{t}$, denoted as $E_{p}\left(p_{t}, R_{t}, u_{t}\right)$ and $E_{R}\left(p_{t}, R_{t}, u_{t}\right)$ respectively, are equal to the optimal solution to this minimization problem.

Let us solve the US household's optimization problem formulated by (1) and (2). Associated with this problem is the Lagrangian

$$
\Upsilon=\sum_{t=0}^{\infty}\left[u_{t} \rho^{t}+\lambda_{t}\left\{\left(1+r_{t}\right) S_{t}+w_{t} L-E\left(p_{t}, R_{t}, u_{t}\right)-S_{t+1}\right\}\right]
$$

The first-order conditions are

$$
\begin{aligned}
& \frac{\partial \Upsilon}{\partial u_{t}}=\rho^{t}-\lambda_{t} E_{u}\left(p_{t}, R_{t}, u_{t}\right)=0 \\
& \frac{\partial \Upsilon}{\partial S_{t}}=-\lambda_{t}+\lambda_{t+1}\left(1+r_{t+1}\right)=0
\end{aligned}
$$

where $E_{u}\left(p_{t}, R_{t}, u_{t}\right)$ denotes the partial derivative of $E\left(p_{t}, R_{t}, u_{t}\right)$ with respect to $u_{t}$. Since we assume a concave felicity function, the second-partial derivative of $E($.$) with respect to u, E_{u u}($.$) , is nonnegative. Thus, the second-order conditions$ are weakly satisfied. ${ }^{4}$ Defining $\theta_{t} \equiv \lambda_{t} / \rho^{t}$, we can rewrite (3) and (4) as

$$
\begin{aligned}
& 0=1-\theta_{t} E_{u}\left(p_{t}, R_{t}, u_{t}\right) \\
& 0=-\theta_{t-1}+\theta_{t}\left(1+r_{t}\right)
\end{aligned}
$$

For given time profiles $\left\{p_{t}\right\}_{t=0}^{t=\infty},\left\{R_{t}\right\}_{t=0}^{t=\infty}$ and $\left\{r_{t}\right\}_{t=0}^{t=\infty}$, and initial condition $S_{0}$, if a value of $\theta_{-1}$ is chosen, the dynamical system which consists of (2), (5) and (6) determines the pair of time profiles $\left\{S_{t}\right\}_{t=0}^{t=\infty}$ and $\left\{u_{t}\right\}_{t=0}^{t=\infty}$. The following lemma states under what conditions the pair is optimal from the viewpoint of the US household.

LEMMA 1: If the value $\theta_{-1}$ is chosen in such a way that the transversality condition

$$
\lim _{t \rightarrow \infty} S_{t+1} \theta_{t} \rho^{t}=0
$$

\footnotetext{
${ }^{4}$ As we shall see later, we specify the felicity function such that it is strictly concave in a relevant region. So, the second-order condition is strongly hold.
} 
holds for given $\left\{p_{t}\right\}_{t=0}^{t=\infty},\left\{R_{t}\right\}_{t=0}^{t=\infty}$, and $S_{0}$, then the pair $\left(\left\{S_{t}\right\}_{t=0}^{t=\infty},\left\{u_{t}\right\}_{t=0}^{t=\infty}\right)$ which is derived from the dynamical system (2), (5) and (6) is an optimal consumption plan of the US household.

Proof: See Appendix 1.

\subsubsection{The Indian household}

Let us turn to the Indian representative household. He solves the following problem

$$
\begin{gathered}
\max _{c_{t}^{*}} \sum_{t=0}^{\infty} U^{*}\left(c_{t}^{*}\right) \rho_{*}^{t} \\
\text { subject to } \\
F_{t+1}^{*}=\left(1+r_{t}^{*}\right) F_{t}^{*}+w_{t}^{*} L^{*}-p_{t} c_{t}^{*}, F_{0}^{*} \text { given, }
\end{gathered}
$$

where $r_{t}^{*}$ is the Indian interest rate. The discount factor $\rho_{*}$ is time-variant and in between 0 and 1 . His felicity depends only on the consumption of wheat, say $U_{*}\left(c_{t}^{*}\right)$. Therefore, his optimization problem is

$$
\max _{F_{t}^{*}} \sum_{t=0}^{\infty} U_{*}\left(\left\{\left(1+r_{t}^{*}\right) F_{t}^{*}+w_{t}^{*} L^{*}-F_{t+1}^{*}\right\} / p_{t}\right) \rho_{*}^{t},
$$

where the discount factor $\rho_{*}$ is constant and between 0 and $1, L^{*}$ the labor endowment of the Indian household, and $w_{t}^{*}$ the wage rate in India at period $t$. Assuming that the felicity function is increasing and strictly concave, we derive the first-order condition

$$
\begin{aligned}
& U_{*}^{\prime}\left(\left\{\left(1+r_{t}^{*}\right) F_{t}^{*}+w_{t}^{*} L^{*}-F_{t+1}^{*}\right\} / p_{t}\right) / p_{t} \\
= & U_{*}^{\prime}\left(\left\{\left(1+r_{t+1}^{*}\right) F_{t+1}^{*}+w_{t+1}^{*} L^{*}-F_{t+2}^{*}\right\} / p_{t+1}\right)\left(1+r_{t+1}^{*}\right) \rho_{*} / p_{t+1}
\end{aligned}
$$

Like Lemma 1, we can prove that for given time profiles $\left\{p_{t}\right\}_{t=0}^{t=\infty},\left\{r_{t}^{*}\right\}_{t=0}^{t=\infty}$ and $\left\{r_{t}^{*}\right\}_{t=0}^{t=\infty}$ and initial $F_{0}^{*}$, the pair of time profile $\left(\left\{F_{t}^{*}\right\}_{t=0}^{t=\infty},\left\{c_{t}^{*}\right\}_{t=0}^{t=\infty}\right)$ satisfying the budget condition and (8) is an optimal plan of the Indian household, if the transversality condition

$$
\lim _{t \rightarrow \infty} F_{t+1}^{*} U_{*}^{\prime}\left(\left\{\left(1+r_{t}^{*}\right) F_{t}^{*}+w_{t}^{*} L^{*}-F_{t+1}^{*}\right\} \rho^{t} / p_{t}=0\right.
$$

is satisfied.

\subsection{Production side}

We shall focus on the case in which labor coefficients satisfy the following inequality

$$
\frac{a_{1}}{a_{2}}<\frac{a_{1}^{*}}{a_{2}^{*}}
$$


which means that US has comparative advantage in the production of wheat; under free trade US exports wheat and India exports computer. (10) itself does not imply that both countries are completely specialized to the production of each of the two goods; either US or India can produce both goods. In what follows, however, we assume that while US produces only wheat, while India is completely specialized to the production of computer. This assumption is justified if $p$ is determined in between $\frac{a_{1}}{a_{2}}$ and $\frac{a_{1}^{*}}{a_{2}^{*}} .5$

Under complete specialization of the prediction of each good, the world outputs of computers and wheat is written as $Y^{*}=L^{*} / a_{2}^{*}$ and $Y \equiv L / a_{1}$, respectively. We also have the two (price) $=$ (average cost) conditions at each period, one for computer and the other for wheat,

$$
\begin{aligned}
1 & =w_{t}^{*} a_{2}^{*} \\
p_{t} & =w_{t} a_{1}
\end{aligned}
$$

It follows that the factor incomes of US and India are

$$
\begin{aligned}
w_{t} L & =\left(p_{t} / a_{1}\right) L=p_{t} Y \\
w_{t}^{*} L^{*} & =\left(1 / a_{2}^{*}\right) L^{*}=Y^{*}
\end{aligned}
$$

\subsection{Market-clearing conditions}

\subsubsection{Credit and rental markets}

We assume that there is no international credit Marietta, while each country has its domestic credit market. The market-clearing condition of the US credit market is $F_{t}=0$, which means that

$$
S_{t}=A_{t}
$$

On the other hand, the market-clearing condition of the Indian credit market is $F_{t}^{*}=0$. It follows from the Indian budget constraint and (11) that the Indian demand function for wheat is written as

$$
c_{t}^{*}=w_{t}^{*} L^{*} / p_{t}=\frac{L^{*}}{a_{2}^{*} p_{t}}
$$

and the Euler condition (??) becomes

$$
\frac{U_{*}^{\prime}\left(\frac{L^{*}}{a_{2}^{*} p_{t}}\right)}{U_{*}^{\prime}\left(\frac{L^{*}}{a_{2}^{*} p_{t+1}}\right) \rho_{*}}=\frac{\left(1+r_{t+1}^{*}\right) p_{t}}{p_{t+1}},
$$

\footnotetext{
${ }^{5}$ As will be clear in the subsequent sections, the steady-state $p$ is independent of labor coefficients $a_{1}$ and $a_{2}^{*}$. Hence, those coefficients can be chosen in such a way that $p$ is in between the two ratios.
} 
which determines the time-profile of the equilibrium interest rate in India $r_{t}^{*}$, given the time-profiles of the price of wheat. (17) means that the marginal rate of substitution between the period- $t$ wheat and the period- $(t+1)$ wheat is equal to their present-value price ratio. Note that this equality implies that the interest rate $r_{t+1}^{*}$ equals the rate of time preference along the stationary state where all prices are kept constant over time. Since $F_{t}^{*}=0$ for each period $t$, the transversality condition becomes

$$
\lim _{t \rightarrow \infty} 0 \times U_{*}^{\prime}\left(\left(1+r_{t}^{*}\right) \times 0+\frac{L^{*}}{a_{2}^{*} p_{t}}\right) \rho^{t} / p_{t}=0
$$

which holds as long as $p_{t}$ converges to a positive constant.

Next, let us consider the rental market of computers. The stock of computers at period $t$ is $A_{t}$. On the other hand, the demand for computer services in the rental market is $E_{R}\left(p_{t}, R_{t}, u_{t}\right){ }^{6}$ Thus, the market-clearing condition at period $t$ is

$$
0=E_{R}\left(p_{t}, R_{t}, u_{t}\right)-A_{t}
$$

Moreover, we assume instantaneous arbitration between the stock of computers and net credit, i.e.,

$$
1+r_{t}=R_{t}+1-\delta
$$

holds at each period $t$.

\subsubsection{The international wheat market}

Finally, let us consider the wheat market. The supply of wheat is $Y$. On the other hand, the US demand for wheat is $E_{p}\left(p_{t}, R_{t}, u\right)$. Since the Indian demand for wheat is, from (16), $c_{t}^{*}=\frac{Y^{*}}{p_{t}}$. Therefore, the international market-clearing condition is

$$
0=Y^{*} / p_{t}+E_{p}\left(p_{t}, R_{t}, u_{t}\right)-Y
$$

\subsection{The dynamic two-country model of international trade}

Putting together the US and Indian households' consumption behavior and market-clearing conditions, we have the dynamic two-country model of international trade as follows.

\footnotetext{
${ }^{6}$ We assume that one unit of computer can supply one unit of services during one period.
} 


$$
\begin{aligned}
A_{t+1} & =\left(R_{t}+1-\delta\right) A_{t}+p_{t} Y-E\left(p_{t}, R_{t}, u_{t}\right) \\
\theta_{t} & =\frac{\theta_{t-1}}{\rho\left(R_{t}+1-\delta\right)} \\
0 & =1-\theta_{t} E_{u}\left(p_{t}, R_{t}, u_{t}\right) \\
0 & =E_{R}\left(p_{t}, R_{t}, u_{t}\right)-A_{t} \\
0 & =Y^{*} / p_{t}+E_{p}\left(p_{t}, R_{t}, u_{t}\right)-Y
\end{aligned}
$$

For a historically given $A_{0}$ and a chosen $\theta_{-1},(23),(24)$ and (25) determine $p_{0}, R_{0}$ and $u_{0}$. Substituting $A_{0}, \theta_{-1}, p_{0}, R_{0}$ and $u_{0}$ into (21) and (22), we derive $A_{1}$ and $\theta_{0}$. Repeating a parallel argument, we obtain a time profile $\left(A_{t}, \theta_{t-1}, p_{t}, R_{t}, u_{t}\right), t=0,1,2,, 3, \ldots$, which depends on what value is chosen for $\theta_{-1}$. Based on Lemma 1, we have the first proposition.

Proposition 1: If $\theta_{-1}$ is chosen in such a way that the time profile starting from $\left(A_{0}, \theta_{-1}\right)$ converges to a steady state where all variables are time-invariant and make sense from economic viewpoint, then the time profile is an equilibrium path.

\section{The existence of steady state}

The steady state of the dynamical system (21)-(25) is a solution to the system of equations

$$
\begin{aligned}
& 0=(R-\delta) A+p Y-E(p, R, u) \\
& 1=\rho(R+1-\delta) \\
& 0=1-\theta E_{u}(p, R, u) \\
& 0=E_{R}(p, R, u)-A \\
& 0=Y^{*}-p\left[Y-E_{p}(p, R, u)\right]
\end{aligned}
$$

The steady-state rental rate is uniquely determined as $\frac{1}{\rho}-1+\delta$. Combining (26) and (29), we obtain

$$
0=(R-\delta) E_{R}(p, R, u)+p Y-E(p, R, u)
$$

Since the expenditure function is linearly homogeneous in $p$ and $R$, the following identity holds

$$
E(p, R, u)=p E_{p}(p, R, u)+R E_{R}(p, R, u)
$$


It follows from (30) that (31) can be rewritten as

$$
\delta E_{R}(p, R, u)=p\left[Y-E_{p}(p, R, u)\right]=Y^{*}
$$

Let $z \equiv \bar{R} / p$, where $\bar{R} \equiv \frac{1}{\rho}-1+\delta$, the steady-state rental rate. Since $E(p, R, u)$ is linearly homogeneous in $p$ and $R$, its partial derivatives, $E_{R}(p, R, u)$ and $E_{p}(p, R, u)$, are homogeneous of degree zero. Therefore,

$$
E_{R}(p, \bar{R}, u)=E_{R}(1, z, u) \text { and } E_{p R}(p, \bar{R}, u)=E_{p}(1, z, u)
$$

Hence, the following lemma is established from (32).

LEMMA 2: Consider the system of equations

$$
\begin{aligned}
E_{R}(1, z, u) & =Y^{*} / \delta \\
E_{p}(1, z, u) & =Y-\left(Y^{*} / \bar{R}\right) z
\end{aligned}
$$

If this system has a solution $(\bar{z}, \bar{u})$, the rest of steady-state variables is uniquely determined as

$$
\begin{aligned}
p & =\bar{R} / \bar{z} \\
\theta & =1 / E_{u}(\bar{R} / \bar{z} \cdot \bar{R}, \bar{u}) \\
A & =E_{u}(1, \bar{z} \cdot \bar{u})
\end{aligned}
$$

\subsection{A mapping from the price ratio $(z)$ into itself}

To derive conditions for the existence of a pair $(z, u)$ that satisfies (33) and (34), we construct a mapping as follows. See Figure 1. For example, take a price ratio $z(=p / \bar{R})$, say $z_{0}$, in the interval $\left[0, \frac{Y \bar{R}}{Y^{*}}\right] . H Q J$ is the graph of the line (34). Then, we derive the intersection of the horizontal line $Q M F$ and the vertical line $E_{R}=Y^{*} / \delta$. Denote the intersection by $M$. The absolute value of the slope of the indifference curve crossing $M, s M s^{\prime}$ is equal to the price ratio $\Theta\left(z_{0}\right)$. By repeating the same procedure for any other $z$ in the interval $\left[0, \frac{Y \bar{R}}{Y^{*}}\right]$, we derive a continuous mapping from price ratio into itself. We denote it by $\Theta(z)$. As is clear from (33) and (34), the steady-state ratio $p / \bar{R}$ is a fixed point of $\Theta(z)$ in the interval $\left[0, \frac{Y \bar{R}}{Y^{*}}\right]$.

The existence of a fixed point depends on how we specify the felicity function $u=U(c, B)$. For example, if it is strictly quasi-concave and homothetic, the absolute value of the slope of indifference curves monotonely increases as we move down to the horizontal axis of coordinates along the vertical line $B=Y^{*} / \delta$. In that case $\Theta(z)$ is monotonely decreasing. If the slope is zero at the intersection of the vertical line $E_{R}=Y^{*} / \delta$ and the horizontal axis of coordinates, then $0=\Theta\left(\bar{R} Y / Y^{*}\right)$ and $0<\Theta(0)$ : the fixed point uniquely exists.

\subsection{Multiple steady states}


In this paper, we specify the felicity function as follows.

$$
U(c, B)=\left\{\begin{array}{c}
\alpha \ln c+\beta \ln B-\gamma c B \text { for } c B \leq \beta / \gamma, c \geq 0 \text { and } B \geq 0 \\
(\alpha-\beta) \ln c+\beta[\ln (\beta / \gamma)-1] \text { for } c B>\beta / \gamma, c \geq 0 \text { and } B \geq 0,
\end{array}\right.
$$

where $\alpha>\beta$. The felicity function is always increasing and strictly concave in $c$ and $B$ satisfying $c B \leq \beta / \gamma$, and non-decreasing and concave in any positive $c$ and $B$. Since concavity implies quasi-concavity, the function satisfies the standard properties as a felicity function. ${ }^{7}$

In contrast to the case of homothetic felicity functions, the mapping $\Theta(z)$ constructed from this felicity function is not monotone. Let us explicitly derive the mapping from the felicity function. Along the vertical line $B=Y^{*} / \delta$ the absolute value of the slope of each indifference curve is

$$
\frac{U_{B}}{U_{c}}=\left\{\begin{array}{c}
\frac{\left[\beta-\gamma\left(Y^{*} / \delta\right) c\right] c}{\left[\alpha-\gamma\left(Y^{*} / \delta\right) c\right]\left(Y^{*} / \delta\right)}, \text { if } c \leq \frac{\beta \delta}{Y^{*} \gamma} \\
0, \text { if } c>\frac{\beta \delta}{Y^{*} \gamma}
\end{array}\right.
$$

Substituting $c=Y-\left(Y^{*} / \bar{R}\right) z$, we explicitly derive the mapping as follows.

$$
\Theta(z) \equiv\left\{\begin{array}{cc}
-\frac{\frac{\delta}{R}\left[z-\frac{\bar{R}}{Y^{*}}\left(Y-\frac{\beta \delta}{Y^{*} \gamma}\right)\right]\left[z-\frac{\bar{R} Y}{Y^{*}}\right]}{\left[z-\frac{R}{Y^{*}}\left(Y-\frac{\alpha \delta}{Y^{*} \gamma}\right)\right]}, & \text { if } \frac{\bar{R}}{Y^{*}}\left(Y-\frac{\beta \delta}{Y^{*} \gamma}\right)<z \leq \frac{\bar{R} Y}{Y^{*}} \\
0 & \text { if } 0 \leq z \leq \frac{\bar{R}}{Y^{*}}\left(Y-\frac{\beta \delta}{Y^{*} \gamma}\right),
\end{array}\right.
$$

where we assume

$$
\frac{\alpha \delta}{Y^{*} \gamma}>Y \geq \frac{\beta \delta}{Y^{*} \gamma}
$$

This assumption means that the graph of the mapping can be depicted as in Figure 2, where

$$
z_{\alpha} \equiv \frac{\bar{R}}{Y^{*}}\left(Y-\frac{\alpha \delta}{Y^{*} \gamma}\right)<0, z_{\beta} \equiv \frac{\bar{R}}{Y^{*}}\left(Y-\frac{\beta \delta}{Y^{*} \gamma}\right) \geq 0, \text { and } \bar{z} \equiv \frac{\bar{R} Y}{Y^{*}}>0
$$

It is bell-shaled with

$$
0=\Theta\left(z_{\beta}\right)=\Theta(\bar{z})
$$

Let us examine under what conditions the graph of $\Theta(z)$ and the $45^{0}$-line have intersections, i.e., fixed points, like $z_{1}(\varepsilon)$ and $z_{2}(\varepsilon)$. First, suppose that $Y$ is equal to $\frac{\beta \delta}{Y^{*} \gamma}$, which is equivalent to $z_{\beta}=0$. Then

$$
\left.\Theta(z)\right|_{z_{\beta}=0}=-\frac{\frac{\delta}{R} z\left[z-\frac{\beta \bar{R} \delta}{\left(Y^{*}\right)^{2} \gamma}\right]}{\left[z+\frac{\bar{R} \delta}{\left(Y^{*}\right)^{2} \gamma}(\alpha-\beta)\right]},
$$

\footnotetext{
${ }^{7}$ See Doi, Iwasa, and Shimomura (2006), where we study the properties of the felicity function is a context of the standard static consumer theory.
} 
the graph of which is depicted by the broken curve. Differentiating $\left.\Theta(z)\right|_{Y=\frac{\beta \delta}{Y * \gamma}}$ with respect to $z$ at $z=0$, we have

$$
\left.\left.\frac{d}{d z} \Theta(z)\right|_{z_{\beta}=0}\right|_{z=0}=\frac{\rho \delta \beta}{\{1-\rho(1-\delta)\}(\alpha-\beta)},
$$

where we use the definition $\bar{R} \equiv \frac{1}{\rho}-1+\delta$. The Inspection of the graph of $\left.\Theta(z)\right|_{z_{\beta}=0}$ in Figure 2 ensures us that if

$$
\frac{\rho \delta \beta}{\{1-\rho(1-\delta)\}(\alpha-\beta)}>1,
$$

which is equivalent to

$$
\frac{(1-\rho+2 \rho \delta)}{(1-\rho+\rho \delta)}>\frac{\alpha}{\beta}
$$

then $\left.\Theta(z)\right|_{z_{\beta}=0}$ has two fixed points, 0 and $z_{0}>0$. It follows from the continuity of the roots of algebraic equation with respect to parameters that at least as long as $Y$ is greater than but sufficiently close to $\frac{\beta \delta}{Y^{*} \gamma}$, then $\Theta(z)$ has two positive fixed points, $z_{1}(\varepsilon)$ and $z_{2}(\varepsilon)$, both of which are in the interval $\left(z_{\beta}, \bar{z}\right)$. We now obtain the lemma as follows.

Lemma 3: If (i) $\alpha>\beta$, (ii) (38) holds and (iii) $\varepsilon \equiv Y-\frac{\beta \delta}{Y^{*} \gamma}$ is positive but sufficiently close to zero, then $\Theta(z)=z$ has two positive real roots, say $z_{1}(\varepsilon)$ and $z_{2}(\varepsilon)$, such that

$$
z_{\beta}<z_{1}(\varepsilon)<z_{2}(\varepsilon)<\bar{z}
$$

and

$$
\lim _{\varepsilon \rightarrow 0} z_{1}(\varepsilon)=0 \text { and } \lim _{\varepsilon \rightarrow 0} z_{2}(\varepsilon)>0
$$

Once a steady-state $z$ is obtained, $c$ and $u$ are uniquely determined by $c=$ $Y-\frac{Y^{*}}{R} z$ and $u=U\left(Y-\frac{Y^{*}}{R} z, \frac{Y^{*}}{\delta}\right)$, respectively. We arrive at the multiple steady-state results.

Proposition 2: Under the conditions on parameters (i), (ii) and (iii) stated in Lemma 2, there are two steady states, $i=1,2$.

$$
\begin{aligned}
R_{i} & =\bar{R}, \quad u_{i}=U\left(Y-\frac{Y^{*}}{\bar{R}} z_{i}(\varepsilon), \frac{Y^{*}}{\delta}\right), \quad p_{i}=\bar{R} / z_{i}(\varepsilon) \\
\theta_{i} & =1 / E_{u}\left(\bar{R} / z_{i}(\varepsilon), \bar{R}, u_{i}\right), A_{i}=E_{u}\left(1, z_{i}(\varepsilon), u_{i}\right)
\end{aligned}
$$




\section{Indeterminacy}

Let us linearize the dynamical system (21)-(25) around a steady state to check the number of characteristic roots which are in between 0 and 1 . The characteristic equation is

$$
\Omega(x) \equiv\left|\begin{array}{ccccc}
\bar{R}+1-\delta-x & 0 & -E_{u} & 0 & Y-E_{p} \\
0 & 1-x & 0 & -\rho \theta & 0 \\
0 & -E_{u} & -\theta E_{u u} & -\theta E_{u R}+\rho & -\theta E_{u p} \\
-1 & 0 & E_{R u} & E_{R R} & E_{R p} \\
0 & 0 & p E_{p u} & p E_{p R} & p E_{p p}+E_{p}-Y
\end{array}\right|=0
$$

As a result of tedious calculations, we get the lemma.

Lemma 4: The characteristic equation is

$$
\Omega(z)=\frac{\{x-(1-\delta)\} \theta}{p} \Gamma(x)=0
$$

where

$\Gamma(x) \equiv(x-1)\left\{\delta E_{R}\left(E_{u u} E_{R R}-E_{u R}^{2}\right)+E_{u}^{2} E_{R R}\right\}-x \rho E_{u}\left(E_{u} E_{R R} \bar{R}-\delta E_{R u} E_{R}\right)$

Proof: See Appendix 2.

According to Lemma 4, one characteristic root is $1-\delta$, which is positive and smaller than one and the other root is the solution to $\Gamma(x)=0$. Inspecting the definition of $\Gamma(x), E_{u u}>0$, and $E_{R R}<0$ imply that $\Gamma(0)>0$. Thus, the other root is in between 0 and 1 , if either $\Gamma(-1)<0$ or $\Gamma(1)<0$. However, we can prove that the felicity function (35) implies that $\Gamma(-1)>0 .{ }^{8}$ Therefore, we have the following lemma.

Lemma 5: The other characteristic root is in between -1 and 1 , if and only if

$$
1-\frac{\delta E_{R} E_{R u}}{\bar{R} E_{u} E_{R R}}<0
$$

A necessary condition for this inequality to hold is $E_{u R}<0$.

Proof: Substituting $z=1$ into (43),

$$
\begin{aligned}
\Gamma(1) & \equiv-\rho E_{u}\left(E_{u} E_{R R} \bar{R}-\delta E_{R u} E_{R}\right) \\
& =-\rho E_{u}^{2} E_{R R} \bar{R}\left[1-\frac{\delta E_{R} E_{R u}}{\bar{R} E_{u} E_{R R}}\right]
\end{aligned}
$$

\footnotetext{
${ }^{8}$ The proof of $\Gamma(-1)>0$ is given in Appendix 3..
} 
Since $E_{R R}<0, \Gamma(1)<0$ if and only if (41) holds. (QED)

Lemma 5 tells us that if the durable consumption good is a "normal good", i.e., $E_{R u}>0$, then (41) does not hold. t follows that while one root is $(1-\delta)$ which is in between -1 and 1 due to Lemma 4 , the other root is outside the closed interval $[-1,1]$. That is, the steady state is saddle-point stable.

Now let us examine under what conditions the inequality (41), i.e., an indeterminacy result, is established. Specifically, we shall show that the inequality (41) holds at the steady state which corresponds to the lower fixed point $z_{1}(\epsilon)$ defined in Lemma 2. As is clear from Figure 2, the mapping $\Theta(z)$ cuts the $45^{0}$-line from below at the lower fixed point $z_{1}(\epsilon)$ and from above at the larger fixed point $z_{2}(\epsilon)$, which means that

$$
\left.\frac{d \Theta(z)}{d z}\right|_{z=z_{1}(\epsilon)}>1 \text { and }\left.\frac{d \Theta(z)}{d z}\right|_{z=z_{2}(\epsilon)}<1
$$

Let us calculate the derivative $\frac{d \Theta(z)}{d z}$. Totally differentiating

$$
\begin{aligned}
E_{R}(1, \Theta(z), u) & =Y^{*} / \delta \\
E_{p}(1, z, u) & =Y-\frac{Y^{*}}{\bar{R}} z
\end{aligned}
$$

with respect to $z, u$ and $\Theta(z)$, we see that

$$
\begin{aligned}
E_{R R} d \Theta(z)+E_{R u} d u & =0 \\
E_{p R} d z+E_{p u} d u & =-\frac{\delta}{\bar{R}} E_{R} d z,
\end{aligned}
$$

from which we derive

$$
\frac{d \Theta(z)}{d z}=\frac{E_{u}}{E_{p u}}\left[\frac{E_{R u} E_{p R}}{E_{R R} E_{u}}+\frac{\delta}{\bar{R}} \frac{E_{R u} E_{R}}{E_{R R} E_{u}}\right]
$$

Because of the linear homogeneity of $E(p, R, u)$ and $E_{u}(p, R, u)$ with respect to $p$ and $R$,

$$
\begin{aligned}
0 & =E_{p R}(1, z, u)+z E_{R R}(1, z, u) \\
E_{u}(1, z, u) & =E_{u p}(1, z, u)+z E_{u R}(1, z, u)
\end{aligned}
$$

has to hold. Taking into these equalities, we can continue from (42) as follows.

$$
\begin{aligned}
(42) & =\frac{E_{u}}{E_{p u}}\left[\frac{\left(E_{u}-E_{u p}\right) E_{p R}}{z E_{R R} E_{u}}+\frac{\delta}{\bar{R}} \frac{E_{R u} E_{R}}{E_{R R} E_{u}}\right] \\
& =1-\frac{E_{u}}{E_{p u}}\left[1-\frac{\delta}{\bar{R}} \frac{E_{R u} E_{R}}{E_{R R} E_{u}}\right],
\end{aligned}
$$

which implies that

$$
\operatorname{sign}\left[\frac{d \Theta(z)}{d z}-1\right]=-\operatorname{sign}\left[1-\frac{\delta}{\bar{R}} \frac{E_{R u} E_{R}}{E_{R R} E_{u}}\right]
$$


It follows that at the lower fixed point $z_{1}(\epsilon)$ where $\frac{d \Theta(z)}{d z}>1$, the term $[1-$ $\left.\frac{\delta}{R} \frac{E_{R u} E_{R}}{E_{R R} E_{u}}\right]$ must be negative. It follows from Lemma 5 that $\Gamma(1)>0$; the two characteristic roots at the steady state are both in between 0 and 1 .

Based on the foregoing analysis, we now derive the main result of this paper.

Theorem: Suppose that the parameters of the model satisfies the following inequalities

$$
\frac{(1-\rho+2 \rho \delta)}{(1-\rho+\rho \delta)}>\frac{\alpha}{\beta}>1 \text { and } \varepsilon \equiv Y-\frac{\beta \delta}{Y^{*} \gamma}>0
$$

If $\varepsilon$ is sufficiently small, then the dynamical system (21)-(25) has two steady state. One of them is saddle, while the other is sink. Thus, if the initial stock $S_{0}\left(=A_{0}\right)$ is in a neighborhood of the latter steady state, then there is a continuum of equilibrium paths which converges to it and which equilibrium path is realized is indeterminate.

Remark 1: Note that the above restrictions imposed on parameters are just a sufficient condition for indeterminacy to hold. Under our specific felicity function, any condition that guarantees the existence of a stationary state is enough to have multiple steady states one of which is a sink. The existence of a steady state implies multiple steady states and local indeterminacy around one of the steady states.

Remark 2: The Jacobian matrices evaluated at the steady states are nonsingular: causality is guaranteed at least in a neighborhood of each steady state.

\section{Concluding remarks}

Let us make a brief remark about implications of our results for international trade theory. It is assumed in the basic trade models like the Heckscher-Ohlin model and the specific factor model that while commodities are freely traded, factors of production are internationally immobile. Unless market distortions like externalities, public goods, government interventions are incorporated into the model, a static trading equilibrium is Pareto-optimal. However, a dynamic equilibrium path which is generated from a multi-country dynamic general equilibrium model of international trade is generally Pareto-suboptimal; for, the rate of marginal substitution between present consumption and future consumption can be different between home and foreign households along the path, if there is no international factor mobility.

This Pareto-suboptimality can generate new theoretical issues in international economics. The indeterminacy discussed in this paper is an example of them. It is our resaerch agenda to study other implications of Paretosuboptimality in international economics. 
If international factor mobility is allowed and/or an international credit markets exist, there is no room for indeterminacy without any other sorce of market distortion; a dynamic general equilibrium path is Pareto-optimal and coincide with the optimal solution of a planner's problem. Thus, from the viewpoint of international economics, indeterminacy is an interesting and important issue. For, international economics has its raison d'être in investigating the intrinsic properties of the world economy which is at an intermediate stage between a segmented world which consists of a set of autarkic economies and a perfectly integrated world. .

\section{Appendix 1. Proof of Lemma 1}

Let us define the period- $t$ indirect utility function

$$
\begin{gathered}
V\left(p_{t}, R_{t},\left(1+r_{t}\right) S_{t}+w_{t} L-S_{t+1}\right) \equiv \max _{c_{t}, B_{t}} U\left(c_{t}, B_{t}\right) \text { subject to } \\
p_{t} c_{t}+R_{t} B_{t} \leq\left(1+r_{t}\right) S_{t}+w_{t} L-S_{t+1}
\end{gathered}
$$

It is well known from duality theory that the following identity holds

$$
\left(1+r_{t}\right) S_{t}+w_{t} L-S_{t+1}=E\left(p_{t}, R_{t}, V\left(p_{t}, R_{t},\left(1+r_{t}\right) S_{t}+w_{t} L-S_{t+1}\right),\right.
$$

Since it is an identity, the partial differentiation of both sides with respect to the "income" term $I_{t} \equiv\left(R_{t}+1-\delta\right) S_{t}+w_{t} L-S_{t+1}$ yields another identity

$$
1=E_{u}\left(p_{t}, R_{t}, V\left(p_{t}, R_{t}, I_{t}\right)\right) V_{I}\left(p_{t}, R_{t}, I_{t}\right)
$$

where $\left.V_{I}\left(p_{t}, R_{t},\left(1+r_{t}\right) S_{t}+w_{t} L-S_{t+1}\right) \equiv \frac{\partial}{\partial I_{t}} V\left(p_{t}, R_{t}, I_{t}\right)\right|_{I_{t}=\left(1+r_{t}\right) S_{t}+w_{t} L-S_{t+1}}$. From (23), (6) and (44), which are evaluated at $t$ and $t+1$,

$\rho\left(1+r_{t}\right)=\frac{\theta_{t}}{\theta_{t+1}}=\frac{E_{u}\left(p_{t+1}, R_{t+1}, u_{t+1}\right)}{E_{u}\left(p_{t}, R_{t}, u_{t}\right)}=\frac{V_{I}\left(p_{t}, R_{t}, I_{t}\right)}{V_{I}\left(p_{t+1}, R_{t+1}, I_{t+1}\right)}=\frac{\rho^{t} V_{I}\left(p_{t}, R_{t}, I_{t}\right)}{\rho^{t} V_{I}\left(p_{t+1}, R_{t+1}, I_{t+1}\right)}$

or

$$
\begin{aligned}
0= & \rho^{t} V_{I}\left(p_{t}, R_{t},\left(1+r_{t}\right) S_{t}+w_{t} L-S_{t+1}\right) \\
& -\rho^{t+1}\left(1+r_{t+1}\right) V_{I}\left(p_{t+1}, R_{t+1},\left(1+r_{t+1}\right) S_{t+1}+w_{t+1} L-S_{t+2}\right)
\end{aligned}
$$

Next, using (23) and ((44), (7) becomes

$$
\lim _{t \rightarrow \infty} S_{t+1} \rho^{t} V_{I}\left(p_{t}, R_{t},\left(1 * r_{t}\right) S_{t}+w_{t} L-S_{t+1}\right)=0
$$

Thus, the pair $\left(\left\{S_{t}\right\}_{t=0}^{t=\infty},\left\{u_{t}\right\}_{t=0}^{t=\infty}\right)$ satisfies both (45) and (46). Applying the standard Mangasarian argument to the present discrete-time framework, we 
can show that, if $V\left(p_{t}, R_{t}, I_{t}\right)$ is concave in $I_{t}$, the pair $\left(\left\{S_{t}\right\}_{t=0}^{t=\infty},\left\{u_{t}\right\}_{t=0}^{t=\infty}\right)$ maximize the US household's discounted sum of utility

$$
\sum_{t=0}^{\infty} V\left(p_{t}, R_{t},\left(1+r_{t}\right) S_{t}+w_{t} L-S_{t+1}\right) \rho^{t},
$$

for time profiles of prices $\left\{p_{t}\right\}_{t=0}^{t=\infty}$, and $\left\{R_{t}\right\}_{t=0}^{t=\infty}$.

Finally we prove the concavity of $V\left(p_{t}, R_{t},\left(1+r_{t}\right) S_{t}+w_{t} L-S_{t+1}\right)$ with respect to $S_{t}$ and $S_{t+1}$. It suffices to prove

$$
V_{I I}\left(p_{t}, R_{t}, I_{t}\right) \equiv \frac{\partial^{2}}{\partial I_{t}^{2}} V\left(p_{t}, R_{t}, I_{t}\right) \leq 0
$$

Partially differentiating both sides of the identity (44) with respect to $I_{t}$, we derive

$$
0=E_{u u}\left(V_{I}\right)^{2}+E_{u} V_{I I}
$$

As we have already stated, $E_{u u}$ is nonnegative, if the direct utility function is concave. It follows that $V\left(p_{t}, R_{t}, I_{t}\right)$ is concave in $I_{t}$. $(Q E D)$

\section{Appendix 2. Proof of Lemma 4}

Let us factorize the characteristic equation

$$
\Omega(x) \equiv\left|\begin{array}{ccccc}
\bar{R}+1-\delta-x & 0 & -E_{u} & 0 & Y-E_{p} \\
0 & 1-x & 0 & -\rho \theta & 0 \\
0 & -E_{u} & -\theta E_{u u} & -\theta E_{u R}+\rho & -\theta E_{u p} \\
-1 & 0 & E_{R u} & E_{R R} & E_{R p} \\
0 & 0 & p E_{p u} & p E_{p R} & p E_{p p}+E_{p}-Y
\end{array}\right|=0
$$

Adding the fourth row multiplied by $R$ and the fifth row to the first row, we have

$$
\Omega(x) \equiv\left|\begin{array}{ccccc}
1-\delta-x & 0 & -E_{u}+R E_{R u} & \bar{R} E_{R R}+p E_{p R} & Y-E_{p}+R E_{R p} \\
0 & 1-x & +p E_{p u} & -\rho \theta & 0 \\
0 & -E_{u} & -\theta E_{u u} & -\theta E_{u R}+\rho & -\theta E_{u p} \\
-1 & 0 & E_{R u} & E_{R R} & E_{R p} \\
0 & 0 & p E_{p u} & p E_{p R} & p E_{p p}+E_{p}-Y
\end{array}\right|=0
$$

Since the expenditure function and its partial derivative with respect to $u$ are both linearly homogeneous in $p$ and $R$, we have

$$
\begin{aligned}
E_{u} & =R E_{R u}+p E_{p u} \\
0 & =R E_{R R}+p E_{p R} \\
0 & =R E_{R p}+p E_{p p}
\end{aligned}
$$


Applying these identities to the first row of (48), we see that

$$
\begin{aligned}
\Omega(x) & \equiv\left|\begin{array}{ccccc}
1-\delta-x & 0 & 0 & 0 & 0 \\
0 & 1-x & 0 & -\rho \theta & 0 \\
0 & -E_{u} & -\theta E_{u u} & -\theta E_{u R}+\rho & -\theta E_{u p} \\
-1 & 0 & E_{R u} & E_{R R} & E_{R p} \\
0 & 0 & p E_{p u} & p E_{p R} & p E_{p p}+E_{p}-Y
\end{array}\right| \\
& =(1-\delta-x)\left|\begin{array}{cccc}
1-x & 0 & -\rho \theta & 0 \\
-E_{u} & -\theta E_{u u} & -\theta E_{u R}+\rho & -\theta E_{u p} \\
0 & E_{R u} & E_{R R} & E_{R p} \\
0 & p E_{p u} & p E_{p R} & p E_{p p}+E_{p}-Y
\end{array}\right|
\end{aligned}
$$

Adding the first row multiplied by $\frac{E_{u}}{1-x}$ to the second row and considering the first-order condition (28) $1=\theta E_{u}$,

$$
\begin{aligned}
= & (1-\delta-x) \\
& \times\left|\begin{array}{cccc}
1-x & 0 & -\rho \theta & 0 \\
0 & -\theta E_{u u} & -\theta E_{u R}-\frac{x \rho}{1-x} & -\theta E_{u p} \\
0 & E_{R u} & E_{R R} & E_{R p} \\
0 & p E_{p u} & p E_{p R} & p E_{p p}+E_{p}-Y
\end{array}\right| \\
= & \{x-(1-\delta)\} \theta \\
& \times\left|\begin{array}{ccc}
E_{u u}(1-x) & E_{u R}(1-x)+x \rho E_{u} & E_{u p}(1-x) \\
E_{R u} & E_{R R} & E_{R p} \\
p E_{p u} & p E_{p R} & p E_{p p}+E_{p}-Y
\end{array}\right|
\end{aligned}
$$

Adding the second row multiplied by $R$ to the third row and applying the above identities again, we can continue

$$
\begin{aligned}
= & \{x-(1-\delta)\} \theta \\
& \times\left|\begin{array}{ccc}
E_{u u}(1-x) & E_{u R}(1-x)+\frac{x \rho}{1-x} E_{u} & \frac{1}{p}\left(E_{u}-\bar{R} E_{u R}\right) \\
E_{R u} & E_{R R} & E_{R p} \\
E_{u} & 0 & -\frac{Y^{*}}{p}
\end{array}\right| \\
= & \frac{\{x-(1-\delta)\} \theta}{p} \\
& \times\left|\begin{array}{ccc}
E_{u u}(1-x) & E_{u R}(1-x)+x \rho E_{u} & \left(E_{u}-\bar{R} E_{u R}\right)(1-x) \\
E_{R u} & E_{R R} & -\bar{R} E_{R R} \\
E_{u} & 0 & -\delta E_{R}
\end{array}\right|
\end{aligned}
$$




$$
\begin{aligned}
= & \frac{\{x-(1-\delta)\} \theta}{p} \\
& \times\left[(1-x)\left|\begin{array}{ccc}
E_{u u} & E_{u R} & E_{u}-\bar{R} E_{u R} \\
E_{R u} & E_{R R} & -\bar{R} E_{R R} \\
E_{u} & 0 & -\delta E_{R}
\end{array}\right|+x \rho E_{u}\left|\begin{array}{cc}
E_{R u} & \bar{R} E_{R R} \\
E_{u} & \delta E_{R}
\end{array}\right|\right] \\
= & \frac{\{x-(1-\delta)\} \theta}{p} \times\left[(x-1)\left\{\delta E_{R}\left(E_{u u} E_{R R}-E_{u R}^{2}\right)+E_{u}^{2} E_{R R}\right\}\right. \\
& \left.+x \rho E_{u}\left(\delta E_{R} E_{R u}-E_{R R} \bar{R} E_{u}\right)\right]
\end{aligned}
$$

\section{Appendix 3. The proof of $\Gamma(-1)>0$}

To prove $\Gamma(-1)>0$, we need to derive the partial derivatives of the expenditure function that appear in (40). Let us recall the definition of the expenditure function. Associated with the minimization problem is the Lagrangian

$$
\Psi=p c+R B+\lambda[u-\{\alpha \ln c+\beta \ln B-\gamma c B\}]
$$

The first-order conditions are

$$
\begin{aligned}
& \frac{\partial \Psi}{\partial c}=p-\lambda\left(\frac{\alpha}{c}-\gamma B\right)=0 \\
& \frac{\partial \Psi}{\partial B}=R-\lambda\left(\frac{\beta}{B}-\gamma c\right)=0 \\
& \frac{\partial \Psi}{\partial \lambda}=u-\{\alpha \ln c+\beta \ln B-\gamma c B\}=0
\end{aligned}
$$

Totally differentiating (49), (50) and (51) with respect to $p, R, u, c, B$, and $\lambda$, we derive

$$
\Xi\left[\begin{array}{c}
d c \\
d B \\
d \lambda
\end{array}\right]=\left[\begin{array}{c}
d p \\
d R \\
d u
\end{array}\right]
$$

where

$$
\Xi \equiv\left[\begin{array}{ccc}
-\alpha \lambda c^{-2} & -\gamma \lambda & \frac{\alpha}{c}-\gamma B \\
-\gamma \lambda & -\beta \lambda B^{-2} & \frac{\beta}{B}-\gamma c \\
\frac{\alpha}{c}-\gamma B & \frac{\beta}{B}-\gamma c & 0
\end{array}\right]
$$

Let $m \equiv \gamma c B$. Making tedious calculations, we see that

$$
\operatorname{det} \Xi=\lambda v(m)
$$

where

$$
v(m) \equiv\left(\frac{\gamma}{m}\right)^{2}\left[\alpha(\beta-m)^{2}+\beta(\alpha-m)^{2}-2 m(\beta-m)(\alpha-m)\right],
$$


which is positive. For,

$$
m^{2}(\beta-m)^{2}-\alpha \beta(\beta-m)^{2}=(m-\sqrt{\alpha \beta})(m+\sqrt{\alpha \beta}),
$$

which is negative if $\alpha>\beta \geq m>0$.

Due to the envelop theorem, $E_{u}=\lambda, E_{p}=c$, and $E_{R}=B$. Moreover, making use of $v(m)$, we derive

$$
\begin{aligned}
E_{u u} & =\frac{\partial \lambda}{\partial u}=\frac{E_{u}}{v(m)}\left(\alpha \beta-m^{2}\right)>0 \\
E_{R R} & =\frac{\partial B}{\partial R}=-\frac{E_{R}^{2}}{E_{u} v(m)}(\alpha-m)^{2}<0 \\
E_{R u} & =\frac{\partial B}{\partial u}=\frac{E_{R}}{v(m)}\left(m^{2}-2 \alpha m+\alpha \beta\right)
\end{aligned}
$$

Moreover, (50) can be rewritten to

$$
E_{R}=\frac{E_{u}(\beta-m)}{R} .
$$

The substitution of those partial derivatives into $\Gamma(-1)$ yields

$$
\begin{aligned}
\Gamma(-1) \equiv & -2\left\{\delta E_{R}\left(E_{u u} E_{R R}-E_{u R}^{2}\right)+E_{u}^{2} E_{R R}\right\} \\
& +\rho E_{u}\left(E_{u} E_{R R} R-\delta E_{R u} E_{R}\right) \\
= & \frac{E_{u}}{R}\left(\frac{E_{R}}{v}\right)^{2}\left[2 \left[\delta ( \beta - m ) \left\{(\alpha-m)^{2}\left(\alpha \beta-m^{2}\right)\right.\right.\right. \\
& \left.\left.+\left(m^{2}-2 \alpha m+\alpha \beta\right)^{2}\right\}+R v(m)(\alpha-m)^{2}\right] \\
& \left.-\rho v(m) R\left\{R(\alpha-m)^{2}+\delta\left(m^{2}-2 \alpha m+\alpha \beta\right)\right\}\right] \\
= & \frac{E_{u}}{R}\left(\frac{E_{R}}{v}\right)^{2}\left[2\left[\delta(\beta-m)\left\{(\alpha-m)^{2}\left(\alpha \beta-m^{2}\right)+\left(m^{2}-2 \alpha m+\alpha \beta\right)^{2}\right\}\right]\right. \\
& \left.+R v(m)\left[2(\alpha-m)^{2}-\rho\left\{R(\alpha-m)^{2}+\delta\left(m^{2}-2 \alpha m+\alpha \beta\right)\right\}\right]\right] \\
= & \frac{E_{u}}{R}\left(\frac{E_{R}}{v}\right)^{2}\left[2\left[\delta(\beta-m)\left\{(\alpha-m)^{2}\left(\alpha \beta-m^{2}\right)+\left(m^{2}-2 \alpha m+\alpha \beta\right)^{2}\right\}\right]\right. \\
& \left.\left.+R v(m)\left[(\alpha-m)^{2}\{(1-\rho \delta)+\rho(1-\delta)\}+\alpha(\alpha-\beta)\right\} \rho \delta\right]\right] \\
> & 0,
\end{aligned}
$$

as was to be proved. In the above calculations, we use the definition of $R \equiv$ $\frac{1}{\rho}\{1-\rho(1-\delta)\}, \alpha>\beta>m(=\gamma c B)>0$ and the assumption $0<\rho, \delta<1 .(Q E D)$

\section{References}

[1] Benhabib, J., and R. E. A. Farmer (1994): "Indeterminacy and growth," Journal of Economic Theory 63, 19-41. 
[2] Benhabib, J., and R. E. A. Farmer (1996): "Indeterminacy and sector specific externalities," Journal of Monetary Economics 37, 397-419.

[3] Benhabib, J., and R. E. A. Farmer (1999): "Indeterminacy and sunspots in macroeconomics," in: J. B. Taylor and M. Woodford eds., Handbook of Macroeconomics 1, 387-448, North-Holland: Amsterdam.

[4] Benhabib, J., Q. Meng, and K. Nishimura (2000): "Indeterminacy under constant returns to scale in multi-sector economies," Econometrica 68, 1541-1548.

[5] Benhabib, J., and K. Nishimura (1998): "Indeterminacy and sunspots with constant returns," Journal of Economic Theory 81, 58-96.

[6] Boldrin, M., and A. Rustichini (1994): "Indeterminacy of equilibria in models with infinitely-lived agents and external effects," Econometrica 62, 323-342.

[7] Chen, Z. (1992): "Long-run equilibria in a dynamic Heckscher-Ohlin model," Canadian Journal of Economics 25, 923-943.

[8] Doi, J., K. Iwasa, and K. Shimomura (2006): "A well-behaved utility function can generate Giffen's paradox, " RIEB D,P, \#181, Kobe University.

[9] Mino, K. (2001): "Indeterminacy and endogenous growth with social constant returns," Journal of Economic Theory 97, 203-222.

[10] Mino, K., K. Nishimura, K. Shimomura, and P. Wang (2005) "Equilibrium dynamics in discrete time endogenous growth models with social constant returns", Kyoto University.

[11] Nishimura, K., and K. Shimomura (2002a): "Indeterminacy in a dynamic small open economy," Journal of Economic Dynamics and Control 27, 271281.

[12] Nishimura, K., and K. Shimomura (2002b): "Trade and indeterminacy in a dynamic general equilibrium model," Journal of Economic Theory 105, 244-259.

[13] Nishimura, K. and K. Shimomura (2005): "Indeterminacy in a dynamic two-country model", forthcoming to Economic Theory.

[14] Nishimura, K., K. Shimomura, and P. Wang (2006): "Production externalities and local dynamics in discrete-time multi-sector growth models with general production technologies", International Journal of Economic Theory 1, 299-312.

[15] Shimomura, K. (2004): "Indeterminacy in a dynamic general equilibrium model of international trade", Chapter 7 in M. Boldrin, B-L Chen, and P. Wang eds., Human Capital, Trade and Public Policy in Rapidly Growing Economies: From Theory to Empirics, Cheltenham: Edward Elgars, 153169. 
[16] Shimomura, K. (1993): "Durable consumption goods and the pattern of international trade", Chapter 5 in: H. Herberg and N.V. Long eds., Trade, Welfare, and Economic Policies: Essays in Honor of Murray C. Kemp, Ann Arbor: Michigan University Press, 103-119. 


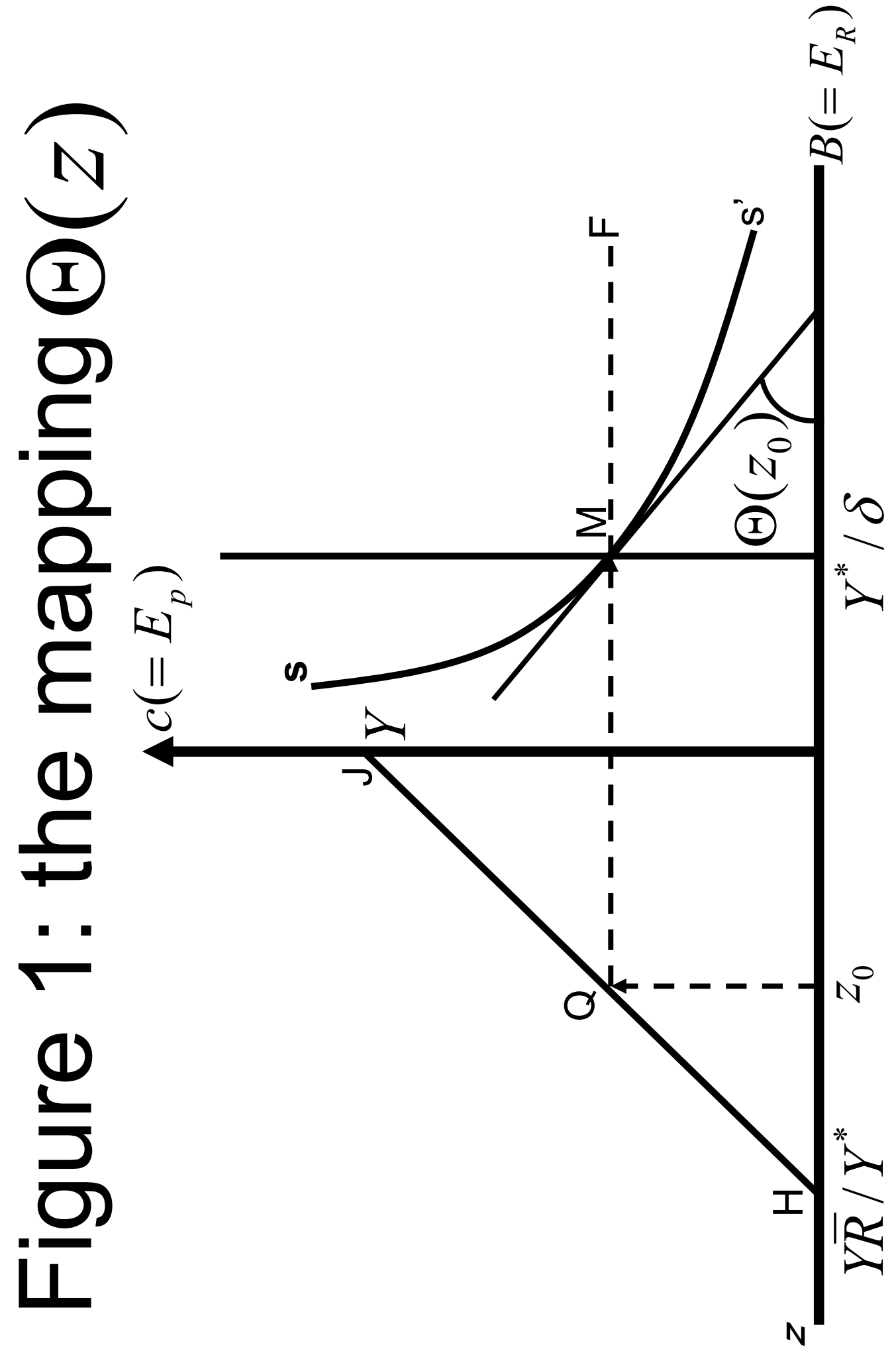




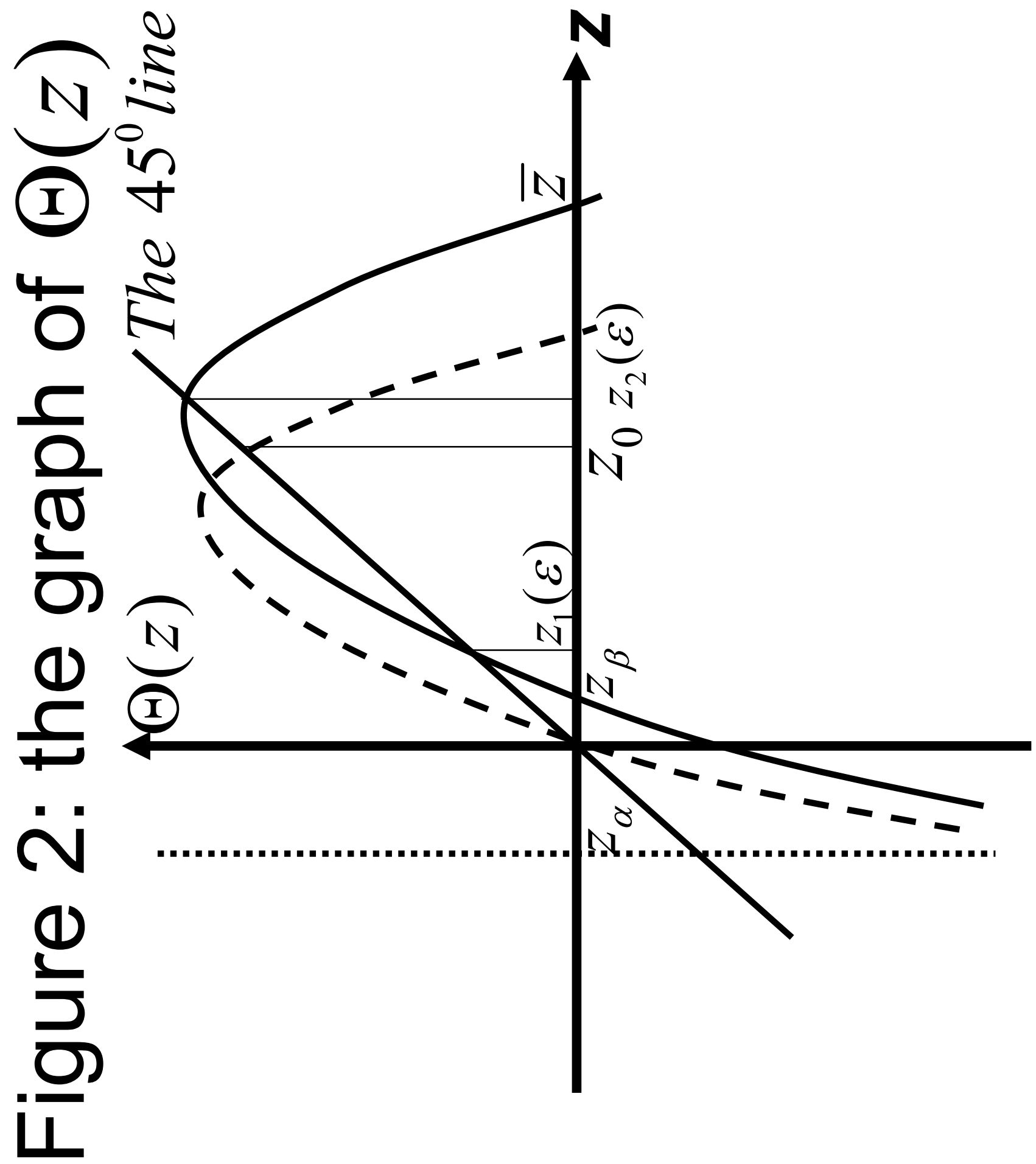

